\title{
Argentina: la ciencia y la ficción
}

PABLO CAPANNA Universidad Tecnológica Nacional, Argentina / pablo.capanna@gmail.com

\section{Resumen}

Dentro de la literatura argentina, la ciencia ficción es un género que cuenta con autores notables pero nunca ha llegado a tener pleno arraigo. Si en países con mayor desarrollo industrial el género ha servido para despertar vocaciones por la ciencia, aquí la relación ciencia/ciencia ficción ha sido menos explícita, aun en el caso de autores con formación científica. Muy distinto fue el proyecto de la revista Más allá que introdujo el género en Argentina durante los años cincuenta. El suyo fue el mayor esfuerzo por abrir el diálogo entre las «dos culturas» y trabajar por su integración.

Palabras clave: ciencia ficción / literatura argentina / revista Más allá / ciencia / divulgación

\section{Science and fiction in Argentina}

\section{Abstract}

Despite having some remarkable authors, science fiction is a genre that has never come to take root in Argentinean literature. At countries with greater industrial development science fiction was useful in awakening vocations for science and technology. In Argentina, the relationship between science and science-fiction has been different, including those authors that have scientific training. Very different was the project of the magazine Más allá that introduced the genre in the fifties, and made the greatest effort to open dialogue between the "two cultures» and integrating them.

Key words: science fiction / Argentinian literature / Más allá magazine / science / popular science

Fecha de recepción: 6/12/2017; fecha de aceptación: 20/3/2018

Para citar este artículo: Capanna, Pablo (2018). «Argentina: la ciencia y la ficción». El taco en la brea 7 (diciembre-mayo), 101-113 Santa Fe, Argentina: UNL. DOI: https://doi.org/10.14409/tb.voi7.7358 
Contra lo que parecería legítimo esperar, los términos técnicos y científicos no siempre se imponen cuando cuentan con la aprobación de la comunidad académica. Lo más común es que entren en circulación por obra de los periodistas, a quienes su profesión obliga a hablar de lo que está en las fronteras del conocimiento. Cuando ni siquiera los científicos atinan a explicarlo de manera llana, los periodistas terminan por aferrarse a alguna expresión llamativa, que a veces hasta puede ser irónica, y esa es la que termina por incorporarse a la lengua común. «Big Bang» nació del malhumor de Fred Hoyle, el primero de los teóricos que se sintió desplazado por la nueva teoría. Los físicos cuánticos, hartos de tener que explicar algo tan ajeno a la experiencia como los quarks, se refugiaron en James Joyce y hablaron, provocativamente, de extrañeza, encanto y sabor, para que nadie se atreviera a preguntar. Al primer púlsar que detectaron, los astrofísicos lo llamaron LGM-1 (la sigla de Little Green Men u «hombrecitos verdes») para evitar que a alguien se le ocurriera hablar de los marcianos.

Si esto ocurre con las ciencias «duras», menos rigor aún podremos esperar de los rótulos que usamos para caracterizar el arte y la literatura. No siempre es fácil explicar en qué sentido hablamos de barroco, romántico, prerrafaelista, fauve o modernista. «Romántico», por ejemplo, nunca deja de cargar con la connotación de «sentimental» y cuesta explicar que es posible aplicarlo a una época o un estilo.

En cuanto respecta a la literatura «culta» contamos al menos con los críticos, quienes se encargan de precisar (o a veces de confundir) los conceptos. Mucho más expuestas a la arbitrariedad comercial están, en cambio, las literaturas genéricas, que necesitan de un rótulo que resulte atractivo para los lectores en busca de evasión. Western, policial, «del corazón», de terror, de aventuras en la selva, de mosqueteros o de piratas, son algunas de las categorías que inventaron los editores en la época en que apareció la cultura de masas.

En estas circunstancias nació el rótulo science fiction, que se usó para designar a esa rama del género fantástico que se nutre del imaginario científico y tecnológico. El rótulo nació de una mera clasificación (ficción + ciencia) y por un momento se lo tradujo al español como «ficción científica». Luego, el hábito acabó por imponer el confuso «ciencia ficción», que sólo sirvió para cargar al género con un estigma que sugiere algo afín a la seudociencia.

Estas decisiones no las tomaron los profesores sino los libreros y antes que ellos los encargados de quioscos de revistas, que tratan de atrapar la imaginación de un lector en busca de entretenimiento. Como suele ocurrir, este sello acabó por hacerse indeleble, de modo que tendremos que aceptarlo tal como es y conformarnos con hacer tan sólo algunas precisiones. Por algo atravesamos una época que es enemiga de las clasificaciones y tiende a diluir todas las diferencias.

Desde sus comienzos, la ciencia ficción convocó a muchos científicos activos y escritores con formación científica, que se sintieron atraídos por la perspectiva de contar con un público amplio, tanto para jugar con esas hipótesis que todavía no podían formular en términos testeables, como para ejercer una suerte de docencia, haciendo divulgación científica.

Con el tiempo, la divulgación llegó a ser una profesión que tiene sus propias reglas. También aparecieron los científicos-estrella que, con la ayuda de algún asesor de estilo, asumían por sí mismos la tarea de divulgar sus ideas más atractivas.

En cuanto a la ciencia ficción misma, la carga científica de los textos siempre osciló entre el polo hard science, donde la trama narrativa era apenas una excusa para exponer conceptos, y el especulativo, donde la fantasía se desprendía de lo científicamente plausible o incursionaba en lo abiertamente imaginario. 
Por amenas y brillantes que resulten para divulgar contenidos científicos, las narraciones de hard science presentan una estructura narrativa pero no deben ser evaluadas estrictamente como obras literarias. Tan sólo cabe juzgarlas en esos términos cuando especulan, con criterios tomados de la ciencia, sobre cuestiones que están en los límites del saber actual y lejos de cualquier proyecto factible.

En el polo opuesto se encuentra el autor que trata de privilegiar el conflicto humano por encima de la ciencia. Todos los conflictos humanos posibles y sus posibles soluciones parecerían ya haber sido inventariados por la crítica en la mayoría de las culturas conocidas. Para que un conflicto en torno a temas eternos como el amor o la muerte pueda ser considerado como ciencia ficción deberá existir algún componente científico o tecnológico que haga a esa historia distinta a cualquier otra versión, sea realista o fantástica, del mismo tema.

El equilibrio entre el polo científico y el estético sólo lo alcanzan los autores de mente científica que incursionan en la ciencia ficción cuando consiguen que el conocimiento aparezca convenientemente metabolizado y juegue algún papel en la trama. Stanislav Lem, un autor que era capaz tanto de escribir sobre la teoría de la robótica como imaginar cuentos morales protagonizados por robots, lo supo exponer de manera ejemplar: «Así como la vaca debe comer pasto para producir leche, yo tengo que leer gran cantidad de textos científicos genuinos (...) pero el producto final, mi escritura, difiere del alimento intelectual tanto como la leche del pasto» (8).

La industria gráfica estadounidense de comienzos del siglo pasado acostumbraba clasificar a los libros como non fiction (algo que iba desde el Manual del Tornero hasta la Historia del Imperio Romano) y fiction, que abarcaba desde las historias de amores contrariados hasta los crímenes inexplicables y las hazañas de los exploradores. En esta última zona también cabían las aventuras en otros planetas y los fatales experimentos de los sabios locos: la science fiction.

El medio cultural norteamericano, en la época en que la ciencia ficción genérica se encargó de codificar toda una rica tradición europea, contaba con una importante comunidad de investigadores y docentes que velaban por la imagen y el prestigio de la ciencia. Desde esa perspectiva, una literatura que lo hacía depender todo de la ciencia y la tecnología era bienvenida para ellos, porque parecía ser útil para despertar vocaciones y reclutar personal técnico.

La situación era muy distinta en Argentina, un país que a pesar de contar con Premios Nobel en ciencia, sólo en tiempos recientes ha comenzado a formular políticas de Estado para la investigación; un país donde las carreras tradicionales siguen siendo las vinculadas al Derecho y la salud. La escasa popularidad de la ciencia hizo que la ciencia ficción argentina siempre contara con un público reducido. Muchos escritores de los que probaron suerte con el género lo hicieron de manera episódica y al margen de los temas con los que más se comprometían. Más allá de unos pocos nombres ilustres y otros tantos rescatables tampoco existió un caudal importante de producción de literaturas genéricas, cuyos lectores por lo general se nutrían de traducciones (Capanna 2007).

\section{Los fundadores}

Los pioneros de la ciencia ficción argentina eran hijos del positivismo y rendían culto a la ciencia: Eduardo. L. Holmberg era zoólogo y Leopoldo Lugones tenía amplios intereses científicos. Pero todos, fieles al espíritu de su tiempo, sentían la atracción del esoterismo y las seudociencias, que sin duda parecían más adecuadas para alimentar la fantasía. La crítica de entonces tenía criterios muy amplios; no estaba especializada y no discriminaba entre las variedades de lo fantástico. Por estas 
razones, los relatos de autores como Lugones o Quiroga solían estar más cerca de Poe, de Hoffmann y de la novela "gótica» que del positivismo cientificista que venía asociado con Jules Verne.

Se suele recordar que Leopoldo Lugones fue uno de los anfitriones de Albert Einstein, cuando el padre de la Relatividad visitó la Argentina. Lugones había conocido a Einstein, Mme. Curie y Henri Bergson en la época en que le había tocado representar a nuestro país en la Liga de las Naciones. Cortésmente, Einstein dijo haber leído el ensayo de Lugones El tamaño del espacio (1921), que estaba lleno de referencias a la Relatividad. Pero a pesar de eso, la ciencia no era lo que estaba en el centro de los intereses de Lugones. Basta repasar sus cuentos de Las fuerzas extrañas (1906) para ver que a pesar de tener una gran curiosidad científica, Lugones se movía con más soltura en el mundo de lo sobrenatural. Algunos de sus textos entrarían hoy en la categoría del Horror, mientras que otros (como «La fuerza Omega» y «La metamúsica») aún logran sorprendernos con sus brillantes intuiciones sobre del poder de los ultrasonidos. Pero en la mayoría de sus cuentos Lugones nos remite a ciencias tan discutibles como la hipnosis, el espiritismo o la homeopatía. Por encima de todo se siente gravitar la Teosofía de Mme. Blavatsky, el credo filosófico al cual Lugones adhirió, al igual que buena parte de los intelectuales de su tiempo. En el «Ensayo de una cosmogonía en diez lecciones» es donde mejor se aprecia la influencia de esa cosmología fantástica que se expone en Isis sin velos y La Doctrina Secreta.

Mientras que el optimismo científico-tecnológico de raíz verniana triunfaba con los utopistas como Julio Dittrich y Pierre Quiroule (Weinberg), el mundo espiritual en el que se movía Lugones era el mismo que frecuentaban los autores de La Novela Semanal, como Ricardo Rojas, Miguel Calvo Roselló o Pedro Angelici (Pierini). Tampoco era ajeno a ese mundo Horacio Quiroga, uno de cuyos últimos libros fue una colección de cuentos fantásticos titulada Más Allá (1935). En uno de ellos, «El vampiro», Quiroga se adelantaba a La invención de Morel de Bioy Casares y hasta a La Rosa Púrpura del Cairo de Woody Allen. Imaginaba a un científico que lograba darle vida a la imagen fantasmal de su estrella de cine favorita. Puesto que el relato pretendía adscribirse a la ciencia ficción y no a lo fantástico sobrenatural, Quiroga le dedicaba nada menos que cinco páginas a fundamentar «científicamente» su historia. Para eso apelaba a unos misteriosos «rayos N1", y citaba a Gustave Le Bon, muy conocido por sus trabajos sobre la "psicología de las masas» y un tanto menos como aficionado a la física. Para entonces los rayos N, a los que también solía invocar Lugones, ya habían sido denunciados como un lamentable error del físico Blondlot, quien se había apresurado a llamarlos «N» en homenaje a la universidad de Nancy. ${ }^{1}$

\section{La era de Más allá}

Sin duda, si hubo un momento en que los lectores argentinos pudieron apreciar la mayor interacción entre la ciencia y la literatura fantástica, fue en las páginas de Más allá, la revista que entre los años 1953 y 1957 dio a conocer lo mejor de la science fiction estadounidense, y ejerció una activa docencia en materia de divulgación científica.

Más allá de la ciencia y la fantasía había nacido en 1953 como una franquicia local de la clásica Galaxy Science Fiction que había fundado H. L. Gold apenas tres años antes en Nueva York. En su mejor momento Galaxy llegó a tener proyección internacional, con sus ediciones británica, francesa, alemana, italiana, holandesa, argentina, finlandesa, sueca y noruega. Algunas de estas publicaciones no tardaron en asumir cierto perfil local, y Más allá lo hizo del modo más activo posible. La versión argentina de Galaxy se llamaba Más allá en homenaje a Horacio Quiroga. 
A pesar de que la revista no daba a conocer la nómina de sus responsables presumimos que detrás de ella, por lo menos al comienzo estuvo Héctor Germán Oesterheld. Más allá publicó un cuento con su firma y otro con el seudónimo Héctor Sánchez Puyol, que llegó a anunciarse en tapa.

La célebre historia de El Eternauta de Oesterheld se gestó en las páginas de Más allá. La nevada mortal estaba en un cuento del periodista Ignacio Covarrubias («Saturnino Fernández, héroe») que apareció en el $\mathrm{n}^{\circ} 27$. El clima paranoico de un mundo invadido por un enemigo insidioso era el mismo que tenía Amos de títeres, la novela de Robert A. Heinlein que había salido en el $n^{\circ} 21$.

Más allá conservaba el equilibrio entre ciencia y ficción que caracterizaba a Galaxy, pero les daba una decidida participación a escritores y científicos argentinos. El material de la revista se componía de algo más de un 50 \% de cuentos y novelas, pero todo el resto eran notas de divulgación científica.

Más allá fue una publicación muy interactiva. Desde el segundo número comenzó a publicar un nutrido correo de lectores que llegaba desde todo el mundo de habla hispana. En el nº 4 lanzó una encuesta destinada a conocer el perfil intelectual de sus lectores, y más adelante comenzó a publicar las opiniones de éstos sobre el material publicado. La revista hizo periódicos balances de su relación con el lector y en todo momento se mantuvo abierta a las sugerencias.

Pronto el volumen de la correspondencia comenzó a crecer tanto que obligó a crear una sección especial para las consultas de carácter científico (Proyectiles dirigidos). Se dice que ésta estuvo a cargo del físico y filósofo Mario Bunge. La revista también acostumbraba a publicar un cuestionario (Espaciotest) donde invitaba a los lectores a poner a prueba sus conocimientos científicos.

Como política editorial, Más allá se proponía asociar las «ficciones científicas» —una vaga categoría que podía incluir desde la poesía de Bradbury y Sturgeon hasta los acertijos de Asimov-con una divulgación de alto nivel. El periodismo científico estuvo presente desde el comienzo, tanto en forma de breves gacetillas al pie de página como de artículos de cierta extensión. En la última etapa de la revista estos llegaron a conformar una sección estable: las Aventuras de la mente.

Esa asociación de la ciencia con la ficción, muy acorde a la ideología desarrollista que se abriría paso con la presidencia de Frondizi, se fue diluyendo a medida que pasaba el tiempo y se sucedían las vicisitudes editoriales. Una vez desaparecida Más allá. la mayoría de sus lectores se fue definiendo más por el gusto literario o por el deseo de evasión que por la búsqueda de información científica. Buena parte del público se mantuvo fiel a esta última y en torno a ellos se generó un activo mercado editorial.

Estábamos a mediados de los años cincuenta, la etapa previa a la irrupción del Sputnik, y la exploración del espacio todavía estaba en el terreno de la fantasía. La iconografía de Más allá tenía el estilo de las revistas norteamericanas de ciencia ficción, aun cuando algunos de los ilustradores fueran argentinos. Abundaban las escenas de la conquista del espacio: las tapas solían exhibir cohetes, estaciones espaciales y visiones del espacio cósmico.

Los temas de los que más se ocupaba la revista también eran los del espacio y la astronáutica. Era habitual que hubiese un artículo de fondo dedicado a ellos, generalmente tomado de Galaxy. En sus primeros nueve números, Más allá publicó, por entregas, el libro La conquista del espacio del alemán Willy Ley, el periodista científico que más hizo para imponer la idea kennedyana del espacio como Nueva Frontera. Las ilustraciones hiperrealistas eran de Chesley Bonestell, que había sido el escenógrafo de la exitosa película Destination Moon (1950). Después vinieron Espacio sin fronteras de Ley y otros ( $\mathrm{n}^{\circ} 26-30$ ), un libro dedicado a ilustrar y promover el proyecto para llegar a 
la Luna con una nave de tres etapas, que Wernher von Braun pensaba venderle al gobierno de los Estados Unidos.

Cuando, el 4 de octubre de 1957, el satélite soviético Sputnik 1 entró en órbita, tomó por sorpresa al mundo entero. Sin embargo, en febrero de ese mismo año Más allá había publicado dos notas sobre los «satélites artificiales», donde sugería que «los rusos mandarán uno bastante antes que los norteamericanos». ${ }^{2}$

Cuatro años antes del Sputnik, el primer número de Más allá ya traía una nota sobre satélites y citaba al Ingeniero Teófilo Tabanera, el pionero argentino del espacio. Más adelante, la revista le daría espacio a sus seguidores, reunidos en torno a una fugaz Sociedad Argentina Interplanetaria. Un lector llegó a sugerir que en la esquina de Hipólito Yrigoyen y Lima, el lugar de Buenos Aires donde hoy se encuentra la estatua del Quijote, se levantara el primer monumento a la Astronáutica, que tendría la forma de un enorme cohete de acero inoxidable.

A lo largo de los cuatro años en que apareció, Más allá siguió publicando por entregas libros como Las edades glaciales de Willy Ley ( $\mathrm{n}^{\circ}$ 19-21), El fin del mundo de Kenneth Heuer $\left(\mathrm{n}^{\circ} 20-25\right)$ y En pos del infinito ( $\left.\mathrm{n}^{\circ} 39-40\right)$.

Quizás el mayor aporte de la revista haya sido el espacio que les brindó a algunos escritores argentinos (que tras el cierre se dispersarían) y a varios científicos profesionales, a quienes les hubiese costado mucho publicar en otros medios.

Entre los hombres de ciencia que probaron suerte como escritores cabe destacar el astrofísico Carlos Varsavsky (1933-1983) Con el seudónimo «Abel Asquini» Varsavsky compuso tres cuentos policiales en clave científica bajo el título Los crímenes del L.I.O. De haberse publicado en el mercado norteamericano una serie como esta habría dado origen a una novela: de ese modo nacieron Crónicas marcianas de Bradbury, Yo, robot, de Asimov y Ciudad, de Simak.

«L.I.O.» era la sigla de un imaginario Laboratorio Internacional Orselec, lleno de científicos envidiosos que se tendían trampas mortales unos a otros. Solían planear el crimen perfecto recurriendo a armas tan poco convencionales como la materia superdensa («Protoníquel», $\mathrm{n}^{\circ}$ 6), los ultrasonidos ( (Nemobius fasciatus», $\mathrm{n}^{\circ}$ 7) o los rayos infrarrojos ( (Nictálopes», $\mathrm{n}^{\circ}$ 8) pero inevitablemente fracasaban porque siempre algún imprevisto lo echaba a perder todo.

Quizás Varsavsky también haya sido el responsable del dossier sobre los platos voladores, al cual la revista le dedicó buena parte del n²4. En esos años, el «fenómeno OVNI» aún no había caído en manos del esoterismo y todavía era común explicarlo como un posible contacto con naves extraterrestres. Así lo prueba el hecho de que Varsavsky aún le siguiera dedicando algunas páginas en su libro Vida en el universo de 1971.

La divulgación que hacía Más allá era de un nivel que hoy quizás sería considerado demasiado elevado para el lector común. Entre los artículos escritos por científicos argentinos, se destacaban los del físico José F. Westerkamp: «El Sol» ( $\left.n^{\circ} 10\right)$ «Las estrellas» ( $\left.n^{\circ} 11\right)$ y «Las Galaxias» ( $\left.n^{\circ} 12-13\right)$. Otros colaboradores científicos fueron Gron Aguirre y Angel Gide (posibles seudónimos), quienes se encargaron de desarrollar la serie La vida en el Universo, que apareció entre el $n^{\circ} 13$ y el 19.

Más allá también le dedicó gran espacio al tema de la energía nuclear (el slogan del momento era Átomos para la Paz) y no menos al peligro de una guerra atómica. Curioso y un tanto risible, si lo vemos en perspectiva histórica, fue un desplegable con «los planos de la locomotora atómica» que apareció en el $n^{\circ} 25$. Más fundamentados fueron, en cambio, los artículos de la serie El átomo a sus órdenes $\left(\mathrm{n}^{\circ}{ }^{37-42}\right)$ que firmaban G. H. Martin, D. Margione y Claude Massot. Probablemente 
estos mismos hayan sido los que compilaron el dossier sobre la bomba de hidrógeno, con énfasis sobre los estragos que hubiese causado en la ciudad de Buenos Aires ( $\mathrm{n}^{\circ} 43,45$ y 46 ). Era una visión apocalíptica que tampoco habrá dejado de influir en la génesis de El Eternauta.

Sin embargo, no todo era física y astronomía en Más allá. La revista también se ocupaba de los temas biológicos y médicos, con artículos como «El misterio de los mellizos» $\left(\mathrm{n}^{\circ}{ }^{16}\right) \mathrm{o}$ «iSe hereda la inteligencia?» ( $\left.\mathrm{n}^{\circ} 12\right)$ firmado por Iginio Alemanes. Más allá ofreció un excelente informe sobre la tecnología y las aplicaciones del microscopio electrónico (Conquistador del micromundo, $\mathrm{n}^{\circ}$ 2021) que difícilmente hubiese llegado a publicarse en cualquier otra revista de circulación masiva.

Uno de los mayores aciertos de Más allá fue un artículo que apareció en agosto de 1954, un año después de la terrible epidemia de polio de 1953. El autor daba cuenta de las investigaciones de Jonas Salk y se preguntaba: «¿Es este el fin de la parálisis infantil?».3 Esta nota apareció diez meses antes de que se anunciara la primera prueba exitosa de la vacuna Salk, que permitió erradicar a la poliomielitis. Con orgullo, la revista lo recordó en su número de junio de 1955. La vacuna, a pesar de todo, sólo llegó a distribuirse en Argentina cuando ya era tarde para evitar una nueva y peor epidemia, la de 1956.

Sólo sabemos del destino que tuvieron los más notorios entre los colaboradores argentinos de Más allá. Mario Bunge fue el primero en ir a radicarse en Canadá, desde donde se construyó una brillante carrera internacional como epistemólogo. Varsavsky tuvo que abandonar el país en 1977 tras el asesinato de su sobrino, e hizo el resto de su trayectoria en los Estados Unidos. A José F. Westerkamp (1918-2014) lo conocimos en la época turbulenta en que los militantes estudiantiles solían acusarlo de ser "cientificista». Luego tuvo un hijo secuestrado en la dictadura y llegó a ser uno de los fundadores del CELS. Casi seguramente, habrá tenido que ocuparse de defender a alguno de aquellos que lo habían increpado años antes.

\section{La era Minotauro}

Al desaparecer Más allá, sus lectores y las nuevas camadas de aficionados al género se volcaron a los libros de Minotauro, que iban a dominar toda la etapa siguiente. La editorial Minotauro, dirigida por Francisco (Paco) Porrúa, es quizás la que más hizo por jerarquizar al género y ampliar su audiencia. Seleccionaba cuidadosamente los textos y los editaba con un nivel de calidad superior al de las publicaciones europeas de entonces.

Con todo, se notaba la ausencia de ese diálogo con el lector que Más allá se había esforzado por construir, y había muchos que parecían añorarlo. Basta repasar la amplia convocatoria con que se lanzó la IIa Convención de lectores y escritores (Mar del Plata, 1968). No sólo se dirigía a los escritores y lectores de ciencia ficción, sino incluía al periodismo científico, los expertos en la temática espacial y hasta los aficionados a los ovnis.

Una figura notable e injustamente olvidada que se dio a conocer en esa circunstancia, fue la matemática Magdalena Mouján Otaño (1926-2005), cuyos mejores textos aparecerían luego en Nueva Dimensión de España. ${ }^{4}$ Los cuentos de Mouján Otaño estaban llenos de humor y en ellos la ciencia estaba presente de una manera tan discreta como las convenciones del género: nada de eso llegaba a interferir con la narración.

Vinculado a Minotauro también estuvo Alberto Vanasco (1925-1993) un escritor que a la hora de acercarse al género ya contaba con fama de vanguardista. De su asociación con el periodista Eduardo Goligorsky nacieron dos volúmenes de cuentos de ciencia ficción (Goligorsky y Vanasco 1967 y 
1976). Vanasco había sido profesor de matemáticas y tenía una formación científica poco común, pero en sus textos también privilegiaba la imaginación, y la ciencia se daba por supuesta. Uno de sus cuentos más celebrados («Post bombum», 1967) se proponía demostrar por el absurdo la precariedad de los conocimientos científicos del hombre de la calle, e imaginaba que serían de nula utilidad a la hora de reconstruir la civilización si sobrevenía esa catástrofe nuclear que todos temíamos.

En cuanto a Eduardo Goligorsky, continuó siendo fiel al género durante años, tanto en calidad de autor como de traductor. En sus relatos los recursos y los clisés de la ciencia ficción clásica eran usados para construir parábolas políticas de actualidad. De la irritación que despertaban en sus destinatarios dan cuenta las amenazas que recibió, esas que acabaron por llevarlo al exilio.

Para entonces había pasado la etapa en que la energía atómica había sido protagonista y los temas de la relatividad y la física cuántica ya estaban llegando al nivel de la vulgarización. Vanasco solía mencionarlas cuando trataba de dar cuenta de la compleja estructura de sus novelas y de sus audacias narrativas. Bastará recordar a otro autor de esos años, Cayetano Ferrari (1943-2016), quien escribió un «Cuento relativista» en el cual jugaba con aplicar las leyes del mundo cuántico a una trama policial, obteniendo resultados francamente surrealistas (Ferrari).

Otro escritor entre todos los que se asomaron a la ciencia ficción en esa época fue Guillermo Boido (1941-2013). Era historiador de la ciencia y profesor de física, pero al igual que sus colegas también tocaba los temas de la ciencia con más ironía y vuelo poético que con intención didáctica.

De los autores que crecieron en torno a Minotauro y se sintieron atraídos por las posibilidades imaginativas que ofrecía el género, casi ninguno tenía más formación científica que la que da la escuela media. Basta recordar que entre los primeros entusiastas de los libros de Minotauro se contaron varios profesores de latín del Colegio Nacional de Buenos Aires, y que uno de los autores más perseverantes de esa generación fue Juan Jacobo Bajarlía, de profesión abogado.

Un fenómeno aparte lo constituyó un grupo de renombrados psicoanalistas que, inspirándose en Theodore Sturgeon, se autoconvocaron para escribir cuentos de ciencia ficción, con los cuales compilaron la antología Ecuación fantástica (Rodrigué y otros). En sus páginas era posible encontrar firmas ilustres, como Abadi, Langer, Rodrigué, Usandivaras, Grinberg, o Rascovsky. Pero, de no haber sido advertido en el prólogo, el lector difícilmente hubiera sospechado cuál era la profesión de los autores.

Quizás toda esta promoción de escritores se haya sentido alentada por el aval de Borges y Bioy Casares, los únicos escritores que manifestaban tener cierto respeto por el género. A Bioy, que tenía intereses análogos a los de Borges y menos contacto aún con la ciencia, le debemos la mejor novela de ciencia ficción argentina, La invención de Morel (1940). Paradójicamente, su gran novela se inspiró en el cine, pero la evolución de la tecnología ha hecho que en una relectura actual parezca casi una metáfora de la realidad virtual.

Angélica Gorodischer, a quien la Fundación Konex consagraría como la mayor exponente del género en nuestro país, solía reivindicar a la ciencia ficción, lo mismo que al cómic y todo lo que fuera ajeno al academicismo. Pero sus lecturas científicas se limitaban al material que traducía para un laboratorio y la literatura que más frecuentaba era otra.

\section{De El Péndulo a Axxón}

A fines de los años setenta, los lectores argentinos de ciencia ficción volvieron a encontrar un polo de atracción en la revista El Péndulo, entre la ficción y la realidad, que dirigía Marcial Souto. En 
su accidentada vida, la revista apareció y desapareció más de una vez. En una de sus mutaciones asumió el formato libro y pasó a llamarse Minotauro (segunda época), con el mismo elenco y otro sello editor. El Péndulo, y en menor medida Minotauro, crearon su propia fórmula, más literaria y con menor orientación científica, conforme al gusto de una nueva generación de lectores.

Con todo, El Péndulo no dejó de publicar por entregas un libro entero de John Sladek (Los nuevos apócrifos) que se burlaba de todas las seudociencias que entonces estaban en boga. La revista Minotauro, por su parte, le dedicó bastante espacio a los temas científicos, tratados ahora con un estilo más bien ensayístico, y alternando con los literarios. Quien esto firma hizo alguna contribución desde su espacio en la revista, para apuntar hacia las nuevas fronteras de la genética y los comienzos de la informática.

Como columnista se dio a conocer Elvio E. Gandolfo, gran conocedor y traductor de ciencia ficción pero también con escaso interés por la ciencia, que pasó por este y otros géneros explorando lo cotidiano del modo más minimalista posible.

Otro autor que saltó a la fama desde las páginas de El Péndulo fue Carlos Gardini, poseedor de esa variada cultura que brinda el noble oficio de traductor. Gardini, quizás el único escritor argentino con una obra dedicada casi por entero a la ciencia ficción, supo hacerse eco de los cambios que en esos años sufrió tanto el género como la misma visión del mundo. Llegó a cosechar varios de los premios UPC, que otorga la Universidad Politécnica de Cataluña sin que eso le valiera aquí el merecido reconocimiento.

El matemático Leonardo Moledo, que para entonces ya era el decano de los divulgadores científicos argentinos, también escribió en El Péndulo pero en sus textos predominaban más la fantasía y el surrealismo que los contenidos estrictamente científicos.

Con el tiempo, pasó la era de las revistas de papel. El público estable se renovó, sin dejar de mantenerse fiel al género, que siguió viviendo en varias revistas digitales, entre las cuales se destaca la legendaria Axxón, de Eduardo Carletti, cuya larga vida superó con creces las de Más allá y El Péndulo.

En las generaciones que se han acercado al género en tiempos más recientes abundan los lectores venidos del mundo de la informática, que por algo es el paradigma por el cual ahora pasan todas las ciencias, tal como lo fue la mecánica en el siglo XVII. Entre los nuevos autores, menos populares por contar con una difusión más restringida, vuelve a haber cierta presencia del sector científico-técnico. Pero lo que más parece gravitar es la propia historia del género con sus convenciones, que despliegan ante los autores noveles toda una gama de modelos consagrados. Entre las figuras que se destacan hay varios con orientación científica, como el periodista científico Alejandro Alonso, premiado en España, el físico Miguel Hoyuelos y el paleontólogo Néstor Toledo, todos competitivos a nivel internacional.

En cuanto al público, se diría que aún hoy, buena parte de los lectores argentinos de ciencia ficción siguen siendo los estudiantes y profesionales de la ciencia y la tecnología. Como antaño, las resistencias al género siguen estando en el sector literario, tanto por los arraigados prejuicios como por obra de un cine espectacular donde los efectos especiales remplazan al talento y logra ahuyentar a más de un lector culto.

La fórmula de Más allá, que aspiraba a tender un puente entre las «dos culturas» nunca volvió a darse, quizás por las mutaciones que sufrió la cultura en más de medio siglo, pero es muy probable que le debamos más de una vocación, y en general una actitud más positiva hacia la ciencia. 


\section{Notas}

1 La historia de los rayos $\mathrm{N}$ se puede leer en mi libro Inspira4 Entre ellos el posiblemente el más conocido sea «Gu ta guciones. Historias secretas de la ciencia (2010).

2 «Últimas noticias acerca del satélite artificial», Más Allá nº tarrak», publicado en Los universos vislumbrados (selección de 44 (febrero 1957), 66.

3 «¿Es este el fin de la parálisis infantil?», Más Allá nº 15 (agosto 1954), 95 .

\section{Bibliografía}

Capanna, Pablo (2007). «La ciencia ficción argentina». Ciencia ficción, utopía y mercado. Buenos Aires:

Cántaro, 263-281.

(2010). Inspiraciones. Historias secretas de la ciencia. Buenos Aires: Paidós.

Ferrari, Cayetano (1967). Con la cara para adentro. Buenos Aires: Instituto Amigos el Libro Argentino.

Goligorsky, Eduardo y Alberto Vanasco (1976). Memorias del futuro. Buenos Aires: Círculo de Lectores.

(1967). Adiós al mañana. Buenos Aires: Minotauro.

Lem, Stanislav (1984). "Azar y orden». Minotauro 7, 95-110.

Pierini, Margarita (Ed. y estudio preliminar) (2009). Doce cuentos para leer en el tranvía. Antología de La Novela

Semanal. Bernal: Editorial de la Universidad Nacional de Quilmes.

Rodrigué, Emilio y otros (1966). Ecuación Fantástica. Buenos Aires: Hormé.

Weinberg, Félix (Ed.) (1976). Dos utopías argentinas de principios de siglo. Buenos Aires: Solar/Hachette. 